\title{
Presentation Technique of Scents Using Mobile Olfactory Display for Digital Signage
}

\author{
Sayumi Sugimoto ${ }^{1}$, Ryo Segawa ${ }^{1}$, Daisuke Noguchi ${ }^{1}$, \\ Yuichi Bannai $^{2}$, and Kenichi Okada ${ }^{1}$ \\ ${ }^{1}$ Faculty of Science and Technology, Keio University \\ Hiyoshi 3-14-1, Kohoku-ku, Yokohama, Kanagawa, 223-8522 Japan \\ \{sugimoto, segawa, noguchi, okada\}@mos.ics.keio.ac.jp \\ ${ }^{2}$ Global Environment Promotion Headquarters, Canon Inc. \\ Shimomaruko 3-30-2, Ohta-ku, Tokyo, 146-8501 Japan \\ bannai.yuichi@canon.co.jp
}

\begin{abstract}
Understanding and attention value of the advertisement will be advanced by adding scents to the digital signage. However, it was difficult to have corresponding one-to-many relationships, movements of users, and precise chronological control of scents. In this study, using mobile olfactory display, we propose the digital signage with scent which takes account of users' movements. The concept of this study is constructing the system having scents, movements, and communication. This system was built by enabling to receive the scent ejection signal from the advertisement, achieve the distance by the image of web-camera, and eject the scents with the strength in accordance with distance, to have the control of scents which accedes to substance of advertisement and positional relationship. As a result of evaluations, the olfactory information was carried down to users with great accuracy. The scents production of the advertisements will be possible with the use of this system.
\end{abstract}

Keywords: Olfactory Information, Olfactory Display, Pulse Ejection, Olfactory Characteristics, Scented Digital Signage.

\section{Introduction}

Information and communication via computers tends to be limited to visual and audio information. However, transmission via all five senses (sight, hearing, touch, smell and taste) has lately attracting much attention [1]. Within those five senses, the olfactory information affects humans largely because the information achieved by the olfactory organs is transmitted to the cerebral limbic system, where governs emotions and memories. Therefore, olfactory information is thought that is has a large importance of providing high effect on humans [2] as well as 3D sound systems and 3D movies.

Trials on transmitting olfactory information together with audio/visual information are being conducted currently in the field of multimedia such as digital signage to 
have more lush expressions, diversified information, and higher sense of reality [3]. However, most of the researches on scents presentation using existing technique had limitations of olfactory devices and thus it was difficult to precisely control the scents chronologically and spatially. In the use of scents on digital signage, the accommodation toward the relationship of one-to-many and the movement of users were challenging because the existing technique could only present the scents to the limited space. Moreover, because only the control of ON and OFF was possible, the accordance of visual information and olfactory information was difficult and thus the setting of scented advertisements in close distance was almost impossible.

In this study, we aim at constructing the scented digital signage system which has been difficult to realize in the past. The concepts of this study are building the system with scents, movements, and communications. The system was built by enabling reception of the scent ejection signal from the advertisement, achievement of the distance by the image of web-camera, and ejection of the scents with the strength in accordance with distance, to allow the control of scents which accedes to contents of advertisement and positional relationship.

This paper is organized as follows: Section 2 introduces related work to our proposal, which is described in detail in section 3. Section 4 contains the descriptions of our devices, and section 5 shows our evaluation results. At last, section 6 concludes this paper.

\section{Related Work}

Trials on the transmission of olfactory information together with audio/visual information are ongoing. Work first started when Heilig developed Sensorama [4] in the 1950s. Sensaroma is the first virtual reality (VR) system that presented olfactory information together with audio/visual information. The effects of the scented movies towards the viewers were studied by Tomono et al $[2,5]$. This study concluded that the understanding of the contents will be increased when the image of the movie and the scents are corresponded to each other. On the other hand, this study also concluded that if the image and the scents are not corresponded, it cause the disturbance and discomfort.

The another example of service that merged scents to movies, Nakamoto et al. [6] designed a smell synthesis device that presents the odor of a virtual object remotely. The system analyzes the smell to be transmitted and presents the analyzed data as the composition ratio of the odor elements. On the receiver side, a feedback control changes the ratio of the odor elements owned by the receiver to reproduce the target odor.

More, NTT Communications used the aroma-emitting device [7] which is able to save the recipes and delivery schedules to emit the scents in accordance with the image of movie from under the seats in movie theatre [8]. They also studied on the difference of passersby's action when the display of the shop has added the scents. As a result, it enhanced the impression of the store and achieved more attention on 
advertisement. The service for cell-phones which provide scents via internet is now constructing. Kaye's article [9] also describes some systems that add scent to web content, and computer controlled olfactory displays such as iSmell [10] and Osmooze [11] are utilized in these systems.

The wearable olfactory display with location sensor had been constructed by Yamada et al [12]. It controls the density of odor molecules; it can present the spatiality of olfaction in an outdoor environment. The olfactory information transmitting system consists of the aforementioned display, a sensing system using three gas sensors, and matching database. The user can experience a real sense of smell while they are walking around through the system by translating obtained olfactory information.

However, the scent presentation techniques of previous works were designed merely to create the sense of experiencing a scent, and they used higher densities and longer presentation times of scent than actually necessary, and thus were hindered by the problem of olfactory adaptation due to scent lingering in the air. To this end, we describe in the next section our proposed technique that makes it possible to experience multiple scents at the same time and which expresses the distance relation between objects as presented by the audio-visual information.

\section{Characteristics of Olfaction}

A fragrance substance is a compound that stimulates the olfactory cells in the nasal cavity. Fragrance substances can be inorganic substances such as hydrogen sulfide and ammonia, although most are organic compounds. It is said that of the approximately two million kinds of organic compounds in existence, about four hundred thousand of these have an odor [13]. However, humans perceive and recognize about five thousand scents routinely. The characteristics of human olfaction are now briefly described.

\subsection{Olfactory Threshold}

The olfactory threshold is the value used as a standard to express the strength and weakness of a scent. Three kinds of values are generally used for the olfactory threshold: the detection threshold, the recognition threshold, and the differential threshold [14], usually expressed in units of mol (concentration) and mass percentage. However, because the olfactory threshold is a measure of the lowest olfactory stimulus intensity at which an individual can perceive scent, this value does not reflect the intensity (strength and weakness) of the scent perceived.

Detection threshold: the smallest density at which scent can be detected and where the user does not need to recognize the kind of smell.

Recognition threshold: the smallest density at which the kind of scent can be recognized, and its value reflects the ability of the user to express quality and characteristics of the scent. 
Differential threshold: the density at which the user can distinguish the strength of a scent, where its value reflects the ability of the user to detect changes in the stimulus and to quantify the change.

Generally, such changes are expressed as the \% change of stimulation quantity of the original. In the case of olfaction, it differs with different kinds of scent, but is in the range of about $13-33 \%$.

\subsection{Adaptation}

Adaptation is the phenomenon where sensory nerve activity is decreased by continuous stimulation by odor molecules. Adaptation itself and the speed of recovery from adaptation differ according to the kind of scent. Adaptation is gradually strengthened over time, but is restored for a short time (3-5 minutes) by eliminating the scent.

In addition, there are various patterns of adaptation, influenced by the kind of scent and recognition factors.

\section{Proposal}

Understanding and attention value of the advertisement are expected to be advanced by adding scents to the digital signage.

Researches on the effect of scents added to the digital signage have already been conducted, but it had no spatial controls over scent presentation. Thus, presentation of multiple scents to the space was difficult. Moreover, because the scents could only be presented to limited space, it was difficult to have corresponding one-to-many relationships and movements of users which can be seen in the system such as digital signage.

In this study, we propose the scented digital signage using the mobile olfactory display which is able to have precise control of scents. This proposal aims at the implementation of scent presentation corresponds to the advertising contents and positional relationship to users moving freely, which leads to the increase in users' understanding, interest and remembrance of contents. More, this proposal constructs the basement of scented media to become widely used.

The requirements to the proposal are described as follows.

- Minimizing olfactory adaptation and scent lingering in the air

- Scents presentation corresponds to contents and positional relationships

- Scents presentation corresponds to visual information

Olfactory adaptation and scents lingering in the air banefully affect the recognition of scents because of the characteristics of olfactory described above, and it causes the failure of scent presentation. Olfactory adaptation prevents the long-term presentation of scents, and scents lingering in the air cause the interfuse of multiple scents. The precise and complicated scents presentation will be possible when those effects were reduced. 
The scents to be presented varies among the advertisements, thus the presentation requirements must be configured with respect to each advertisement. In other words, the structure, which enables to set kinds, strength, and ambit of the scents and eject them in accordance with users' movement, is needed. Also, the sense of perspective, whether the user is getting close to or moving away from advertisement, needs to be presented in order to tell the source of scents easily. These complicated scent presentation delivers the contents clearly and is expected to further the attention values and understanding of advertisement.

At last, olfactory information must be corresponded to visual information. When these information disaccord, users will sense the discomfort.

The methods to fulfill those requirements are described with details in the following sections.

\subsection{Scents Ejection Policies}

In this study we set the policies in order to realize the scent presenting corresponds to human's olfactory characteristics. The policies are:

1. Eject the $0.1 \mathrm{sec}$ scents pulse with the interval of $1.3 \mathrm{sec}$.

2. Eject one scent at a time

3. Amount of scents are increased by double series

4. Eject the scents in accordance with visual information

Policy 1 is to reduce the effects of olfactory adaptations and scents lingering in the air. Ejecting the scents by the pulse minimize the lingering scent diffuse in the air and diminish the olfactory adaptation by discrete stimulus [15].

Policy 2 is to handle multiple scents. Ejecting the multiple scents at the same time causes the interfusion and scents become unrecognizable. However, due to the results of the separable recognition threshold of scents, the kinds of scents could be recognized if the interval of pulses is more than $1.2 \mathrm{sec}$. [16]. Thus, by applying Policy 1 and Policy 2, we assumed that the multiple scents presentation become possible.

Policy 3 is for presenting the changes of scents' strength. Our previous research [17] showed that increasing the amount of scents in double series enables to present the sense of perspective. In this study, we divided the strength of scent into some levels and the amount of scents is doubled when the level of strength is increased. Here, we call the strength of the scents at the level 1 "base ejection amount". When the strength level is indicated as $\mathrm{S}$ and the base ejection amount as $\mathrm{E}$, the total ejection amount is represented as follows.

$$
\mathrm{B}=\mathrm{E} \cdot 2^{(\mathrm{S}-1)}
$$

Policy 4 is for corresponding the visual information and olfactory information. Here, five senses of human are related to each other and the understanding and reality increases when corresponded information is given.

Ejecting the scents by following those 4 policies, it is able to present the scents based on olfactory characteristics of human because those policies are to reduce the effects of olfactory characteristics and correspond the changes of multiple scents' 
strength. Herewith, it is able to present the scents in accordance with users' movements and contents, which was difficult to realize with traditional scent presenting technique.

\subsection{Scent Presentation Corresponds to Contents and Positions}

In this section, we describe the presentation technique in accordance with contents and position in detail.

The concept of proposal is constructing the system with scents, movement, and communication. It receives the scent ejection signal from the advertisement and achieves the distance by the image of web-camera to present the scents which accedes to substance of advertisement and positional relationship. Traditional scented digital signage had not supposed the strengths and kinds of scents to change, which led them to present one kind of scent to the space. However, there is the need to transmit the information about the scent and the source of the scent to users in order to change the presenting scents in accordance with users' movement.

In this study, the advertisement communicates with users via the wireless device to send the information of scents. The scent information and scent ejection order are sent from the advertisement at regular intervals. The AR markers were used to discern the source of scent by using the web camera.

Figure 1 shows the implementation image of the system. As shown in the figure, the devices are set on the users and the contents and distance information is achieved. Figure 2 is the image of the experimental environment. The markers labeled A, B and $\mathrm{C}$ and were put on the wall as if it were the advertisement, and is sending the scent ejection order at interval of $1.3 \mathrm{sec}$. These orders include 4 kinds of information. In this study, according to 4 scent presenting policies, the width of pulse ejection is set at $0.1 \mathrm{sec}$.

- Kind of scent represent the advertisement

- Kinds of scents that each marker represents

- Strength of each scent

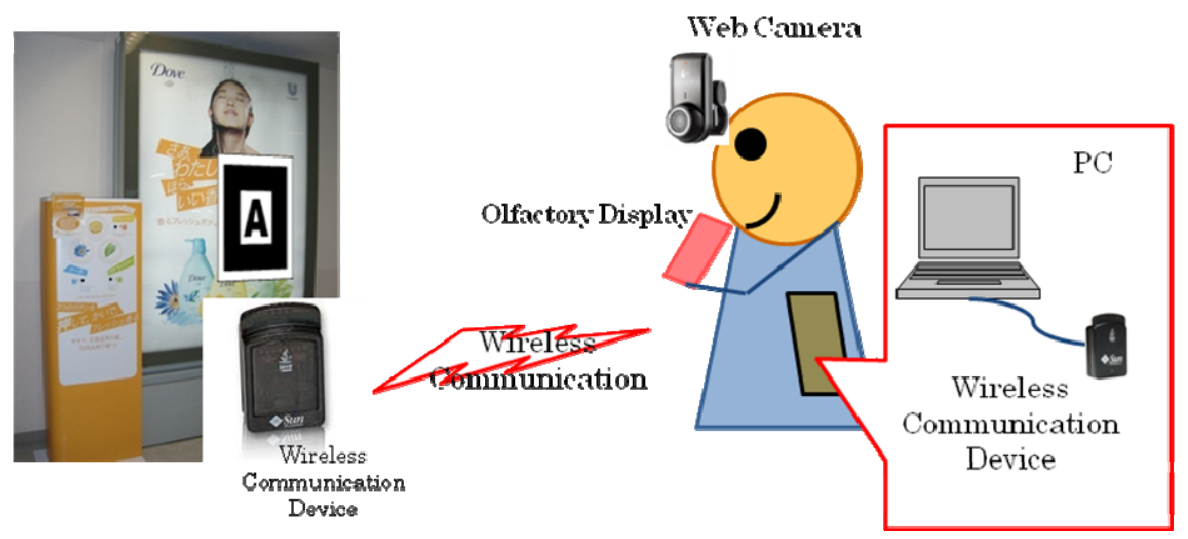

Fig. 1. Implementation Image 


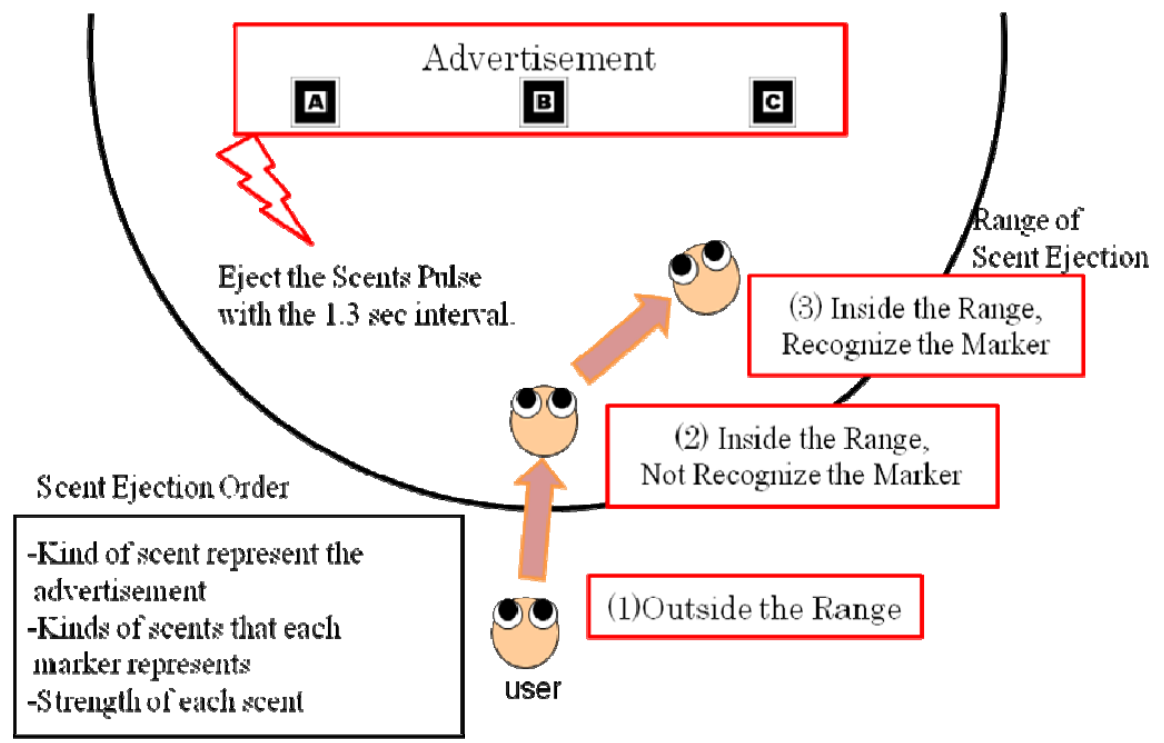

Fig. 2. Image of the experimental environment

The state (1) in figure 2 shows the case when user is outside the range of scent ejection, and the scent is not ejected.

The state (2) in figure 2 shows the case when user is inside the range of scent ejection but cannot recognize the marker, and the scent of advertisement is ejected. The purpose of this background scent is to increase the attention value of advertisement.

The state (3) in figure 2 shows the case when user is inside the range of scent ejection and able to recognize more than on marker. In this case, the scent is ejected by following the ejection priorities. Following the ejection priority and presenting the scent corresponds to the marker that user is looking at increase the understanding of contents because of the synergic effect of visual and olfactory information.

\subsection{Ejection Priorities}

In order to have corresponding between the visual information and olfactory information, we set the ejection priorities in this study. The olfactory priorities are the values range from 0 to 5 and contained by each marker and the background. The ejection priorities are update every time when it receives the scent ejection orders from the image of web camera. Then, the scent that holds highest ejection priority is ejected. When 2 or more scents have the same ejection priority, the scents of marker closest to the center is ejected.

The weight of the ejection priorities are as follows, and the weight and the percentage of the time that marker was detected are multiplied to calculate the updating ejection priority. 
- center: +3

- in the range out of center : +2

- out of range: -1

Also, the ejection priority of background scent is set at 2.5, which is half of the maximum ejection priority. It is decrease by 2 when the scent is ejected.

Using those ejection priorities, it is able to present the scent of marker that the user is looking at.

\subsection{Scent Strengths and Scent Range}

We devised that the scent is ejected when the user looks at the marker. Here, to present the sense of perspective, the changes in range and strength of scents are required.

We supposed that the stronger scent has the larger range, and it is reflected on scent ejection order. The range is calculated from each of the markers. We indicate the base scent strength level as $S$, the scent strength decreased by the distance as S', constant value as $\mathrm{K}$, and distance as $\mathrm{D}$ in the formula. Here, $\mathrm{S}^{\prime}$ is in range of 1 to $\mathrm{S}$.

$$
\mathrm{D}=\mathrm{K} \cdot \sqrt[3]{2^{(S-S)}}
$$

We took the cubic root because the scent chemicals diffuse three-dimensionally. Figure 3 shows the image of maximum ejection when base scent strength $\mathrm{S}$ is equal to 1 and 4.
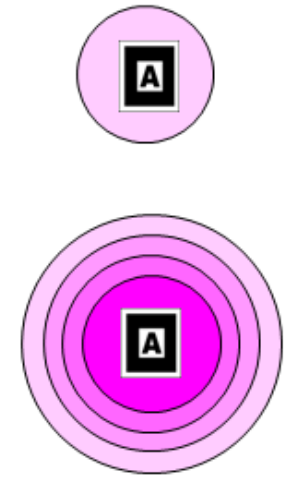

StrengthLevel: 1

Ejection Amount $=E$

$\mathrm{D}=\mathrm{K}$

StrengthLevel : 4

Ejection Amount $=E \times 2^{3}$

$\mathrm{D}=\mathrm{K} \times \sqrt[3]{2 \times 2 \times 2}$

Fig. 3. Ranges and Strength of Scents

\section{Devices}

\subsection{Mobile Olfactory Display}

Figure 4 (a) shows the mobile olfactory display, and Figure 4 (b) shows the image of wearing the display. 

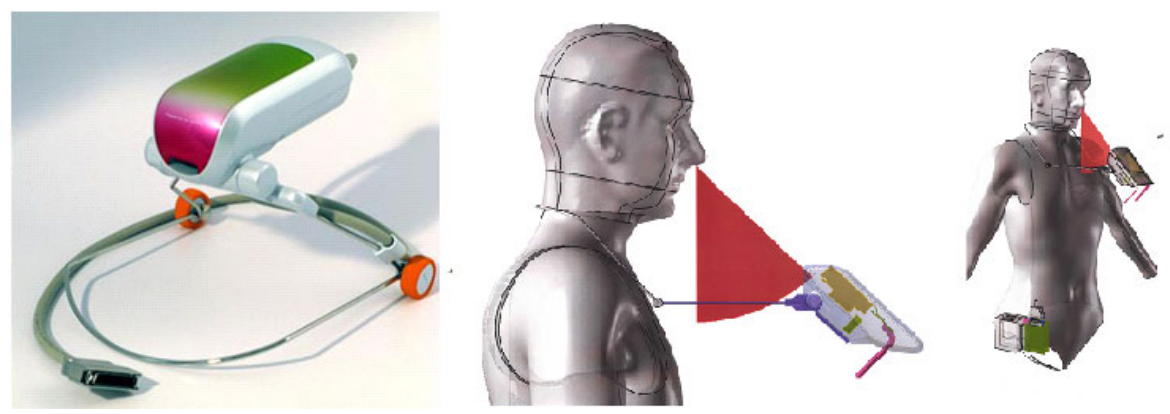

Fig. 4. (a) Mobile Olfactory Display (b) image of wearing display

This display uses the thermal method used in ink-jet printer to produce a jet which is broken into droplets from the small hole in the ink tank. The thermal method installs a heater in a part of the slight narrow tube filled with ink. In order to emit ink, bubbles are formed in the ink by instantaneous heating. This method is advantageous for miniaturization because the structure is simple.

An ejection heater is installed in the liquid route which continues to the exit hole. The ejection heater runs hot so that bubbles are generated in scent in the liquid route. Through the generation of pressure, the scent head emits scent from the exit hole. The scent fills the liquid route and liquid room behind. The scent in the liquid room is supplied along the supply route from the scent tank. By the micro fabrication technique, the production technology of the head which has more 100 exit holes was established. This head can emit scent of several pico-liters.

The unit average ejection quantity from one small tank is $4.7 \mathrm{pl}$ and one large tank is $7.3 \mathrm{pl}$. It was confirmed to be approximately constant without depending on the residual quantity of ink on examination. In addition, the user can set the ejection number of times from one hole in $100 \mathrm{msec}$ to $1-150$ times, which we denote the "volume". By setting the volume, the ejection control is possible for a unit of 667 micro sec.

The display can set up 1 scent-ejection head. This head can store three small tanks and one large tank, thus this display can contain 4 kinds of scents maximum. There are 127 minute holes in the head connected to the small tank and 255 minute holes in the head connected to the large tank. Moreover, the display can emit scent from multiple holes at the same time, so the ejection quantity is adaptable to 0-127 (small tank), 0-255 (large tank).

Also, the display is equipped with a fan and the maximum wind velocity is $1.0 \mathrm{~m} / \mathrm{sec}$. The scent presentation hole is a rectangle of $2 \mathrm{~cm}$ length and $6 \mathrm{~cm}$ width.

The battery is designed to be hanged around the waist, and the mobile olfactory display is designed to wear around the neck. The angle of the display is adaptable to 0-45degrees.

Because the quantity of scents emitted is small and diffuse quickly, this invented olfactory display is suited for personal use. 


\subsection{SUN Spot}

We used SunSPOT from Sun Microsystems as a wireless communication device. SunSPOT uses Zigbee to transmit the information without any losses within range of 10meters. We first placed the SunSPOT remote sensor on advertisement and connected the SunSPOT base station to the laptop. This enables the communication between the advertisement and the user. Also, when the web camera could not recognize the AR marker, the distance between advertisement and the user is calculated by RSSI (Received Signal Strength Indication).

\subsection{Web Camera}

We used the web camera named 2-MP Portable WebcamC905m from Logicool. The frame rate of this camera is $30 \mathrm{fps}$ at maximum, and the angle of view is 76 degree. This camera is used to recognize the AR marker to measure the direction and distance to advertisement of user. The distance was calculated from the number of the height pixels of the marker.

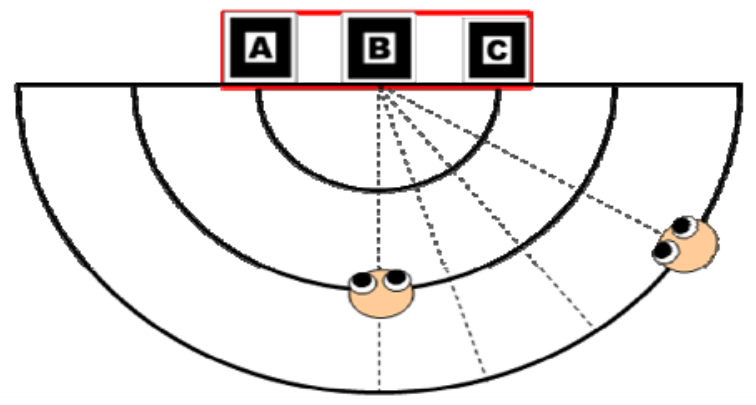

Fig. 5. Experimental environment for Experiment 1

The participants were asked to mount all hardware for two reasons. The first reason is our focuse on presenting the scents of the object that the user is looking at in the personal space. In order to find the object looked at by the user, the webcam must be worn by the user, and to present the scent personally, the olfactory display must be worn by the user to avoid the presentation of scents to non-users. The second reason is the mechanism of the devices. The olfactory display we used must be connected to the computers to be controlled, and so does the receiver of the wireless communication device to receive the signal from the device set on the advertisement.

\section{Evaluation and Discussion}

\subsection{Evaluation on Achieving the AR Markers}

The purpose of this experiment is to evaluate the acquisition accuracy of the marker and to consider the range of implementing proposed system. If the marker that user is 
looking at and the marker that web camera achieved had large difference, it is difficult to observe Policy 4. The experiment was conducted with 11 participants.

Figure 5 shows the overhead view of experimental environment. As shown in Figure 5, the AR makers labeled A, B, and C and were placed on the wall, as if it is an advertisement, with equal distance among them. Then, the participants were asked to look at the marker which was indicated, and evaluated the image from web camera to see whether the camera catches the indicated marker at the center. At the same time, the information about which marker is retrieved by the webcam is shown on the display of computer. By calculating the accuracy, the data is collected.

Here, the positions of the participant were fixed to 12 points, and the experiment was conducted by changing the distance from wall to the user, angle, and distance between the markers. Each of the conditions is as follows.

- Distance to the marker; $1 \mathrm{~m}, 2 \mathrm{~m}, 3$.

- Angle related to the marker: 0, 20, 40, 60,

- Distance between markers; $1.0 \mathrm{~m}, 0.5 \mathrm{~m}$
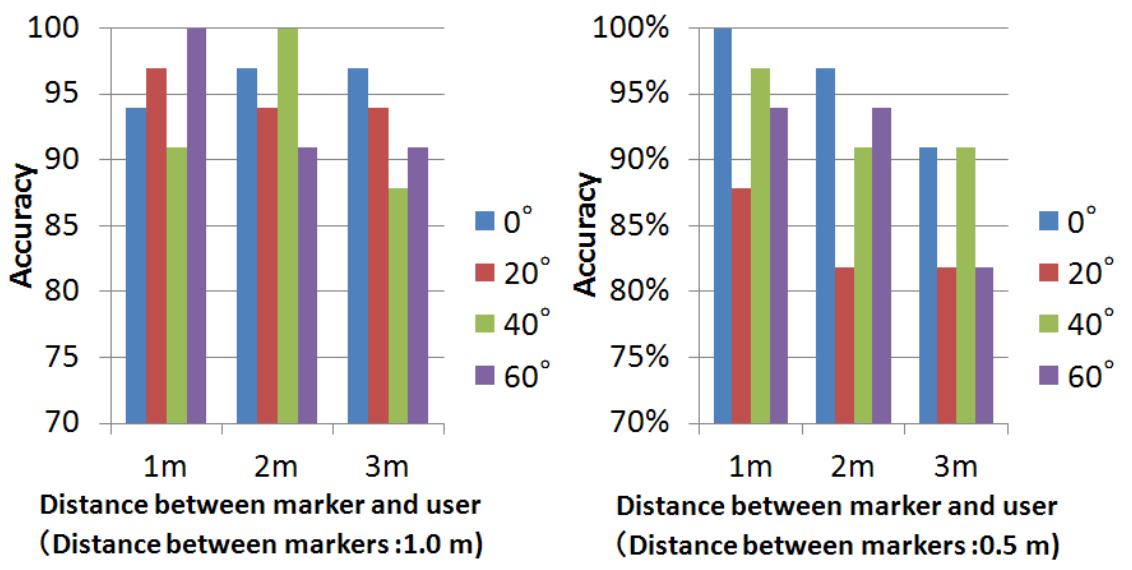

Fig. 6. Result of Experiment 1

The result is as shown in Figure 6. The $\mathrm{x}$-axis is the distance between user and markers, and the $y$-axis is the accuracy of setting the marker in the center of image.

The result shows that the accuracy is more than $80 \%$ for all the conditions. Also, it indicates that the accuracy declines as the user move away from the wall.

Then, the data according to distances between markers are analyzed using two-way ANOVA (distance to the marker, angle related to the marker). The analysis showed that there were no significant differences when the distance of markers were $1.0 \mathrm{~m}$. It means that the accuracy of setting the marker in the center of image is almost same at any position. On the other hand, there were significant difference when the distance of markers were $0.5 \mathrm{~m}(\mathrm{p}<0.5)$. However even, the worst accuracy of setting the image in center was $80 \%$, thus the effect on implementation is small. 


\subsection{Evaluation on Transmitting Olfactory Information}

The purpose of second experiment is to evaluate whether the olfactory information is transmitted to the user accurately and is contributed to find the source of the scent. The experiment was conducted to 14 participants.

10 AR markers were put on the wall with equal distance among them, and the level 1 scent of peppermint was presented all the time as a background scent. Under this environment, participants were asked to move around freely to find the marker with scent of lavender or lemon. Then the ratio of collect answers were calculated and analyzed. The experimental conditions were as follows.

1. 1 scent (distance among marker is $20 \mathrm{~cm}$ )

2. 2 scent (distance among marker is $20 \mathrm{~cm}$ )

3. 1 scent (distance among marker is $40 \mathrm{~cm}$ )

4. 2 scent (distance among marker is $40 \mathrm{~cm}$ )

Table 1. The strength level scents and their range

\begin{tabular}{|c|c|}
\hline Strength Level & Range of Scents (m) \\
\hline 1 & 3.1 \\
\hline 2 & 2.5 \\
\hline 3 & 2.0 \\
\hline
\end{tabular}

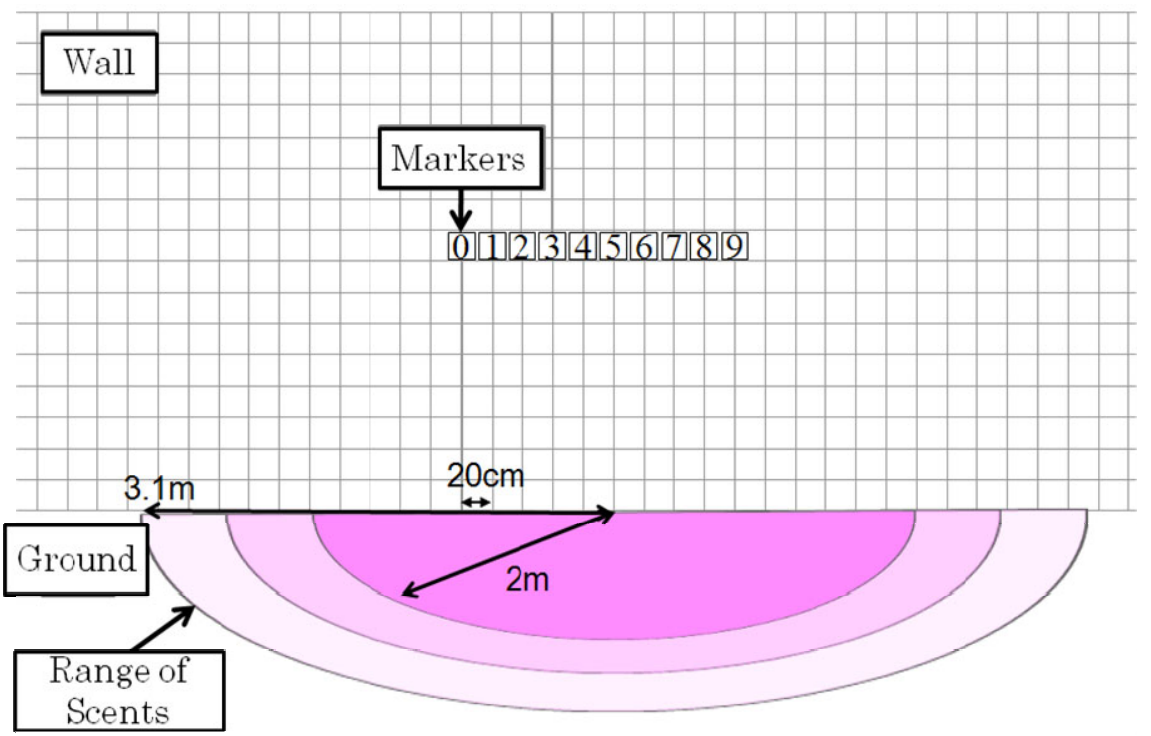

Fig. 7. Image of the range and perspective of scent from obliquely upward 
The scent of lavender (strength level 3) was used when only one scent is presented, and the scents of lavender (strength level 3) and lemon (strength level 3) were used when two scents are presented. For all experiment, each of the scent of lavender or lemon was put on one out of 10 AR markers.

The levels of the scents were changed in accordance with the distance from the marker as shown in Table1. Figure 7 is the image of the range and perspective of scent from obliquely upward when experimental condition 1, when the scent of lavender was put on the AR marker labeled 5 is conducted.

The result is shown in Figure 8. The y-axis represents the percentage of correct answers of finding the scent source. From this graph, it is implied that larger the distance among markers, larger the percentage of correct answers.

Then, Then, the data are analyzed using two-way ANOVA (distances between markers, number of scents presented). The analysis resulted that the distance among markers had the significant difference $(\mathrm{p}<0.01)$. It indicates the difficulty of finding the source of scents by olfactory when the AR markers were placed in the distance of $20 \mathrm{~cm}$. On the other hand, it is accurate to find the scents source when the AR markers are placed with the distance of $40 \mathrm{~cm}$.

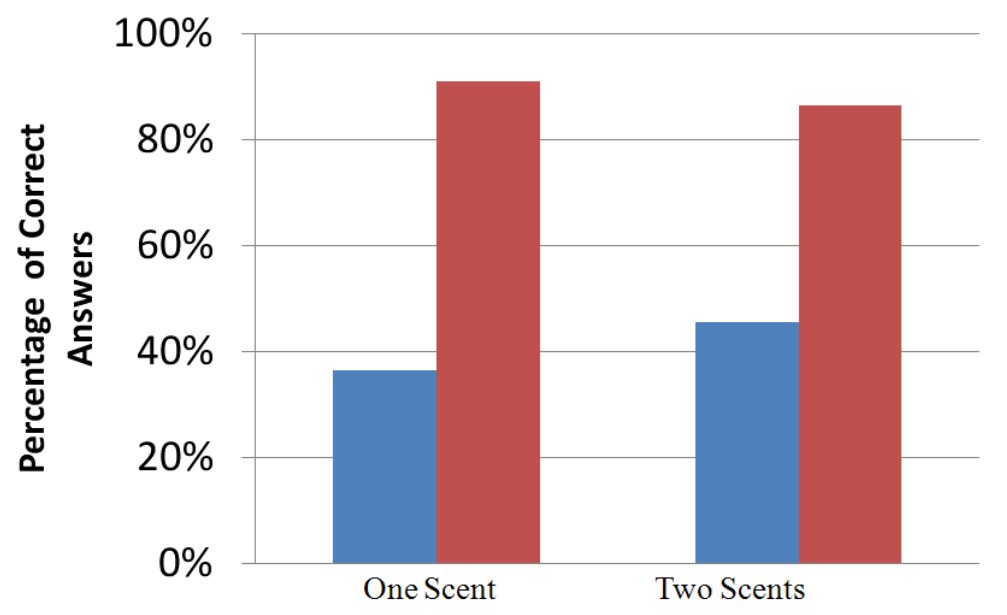

Fig. 8. Results of Experiment 2

Moreover, there was no significant difference based on the numbers of scents $(\mathrm{p}>0.05)$. This means that the presentation and transmission of multiple scents is possible with the same environment which single scent is presented.

From the overall result of this experiment, it can be concluded that transmission of olfactory information and searching of scent source are possible when the AR markers were placed with the distance of $40 \mathrm{~cm}$. Also, when the distances of markers were $20 \mathrm{~cm}$, it can be assumed that the accuracy will be increased when visual information is added. 


\section{Conclusion}

Studies on transmitting information via five senses are currently conducted to present more realistic experiences in the field of multimedia. Presenting the olfactory information together with visual and audio information increases understanding and attention value of the contents, and thus the studies on adding the scents to digital signage has been conducted.

However, many of the researches have the limitation of olfactory displays, and it was difficult to control the scents chronologically and spatially. Moreover, because of the control difficulties, presenting more than one scents from the advertisement was almost impossible.

In this study, to overcome the scent control difficulty, we proposed the scented digital signage using mobile olfactory display. This system not only adds multiple scents on one advertisement but also present the scents in accordance with users' movement, advertisement contents, and spatial relationship.

We first proposed 4 policies of scent ejection based on our previous studies. Then we implemented the system with scents, movements, and communication observing the policies. This presents the scents according to the user's movement and advertisement's contents.

From the results of experiments, we verified the ability of this system. This system is able to present multiple kinds of scents and to find the source of scents only by using olfactory information when the AR markers were more than $40 \mathrm{~cm}$ apart.

As the future work, the appliance of moving picture ads and the revision of policies in accordance with the moving picture ads as well as the experiments with multiple users.

Acknowledgement. This work is supported in part by a Grantin-Aid for Scientific Research(B) ,2011, 23300049 from the Ministry of Education, Culture, Sport, Science and Technology in Japan.

\section{References}

1. Kim, J.-D., Kim, D.-J., Han, D.-W., Byun, H.-G., Ham, Y.-K., Jung, W.-S., Park, J.-S., Oh, S.-K.: A Proposal Representation, Digital Coding and Clustering of Odor Information. Computational Intelligence and Security 1, 872-877 (2006)

2. Tomono, A., Yamamoto, S., Utsunomiya, M., Ikei, D., Yanagida, Y., Hosaka, K.: Effect that the Image Media with Scent Gives to Contents Understanding. In: Human Interface Symposium 2004, pp. 249-254 (2004)

3. Hirose, M., Tanigawa, T.: Information and Communication of Odor. Number 21 in Aroma Science Series. Fragrance Journal Ltd. (2007)

4. Retrofuture: Sensorama's pre-virtual reality, http: / / www.retrofuture.com/sensorama.html

5. Tomono, A., Kanda, K.: Approach of Improving Contents Quality by Smell Addition to Image. Journal of Institute of Electrical Engineers of Japan 128(12), 478-486 (2008) (in Japanese)

6. Nakamoto, T., Nakahira, Y., Hiramatsu, H., Oriizumi, T.: Odor Recorder Using Active Odor Sensing System. Sensors and Actuators B 76, 465-469 (2001) 
7. NTT Communications to Test Aroma-Emitting Digital Signage, http: / /www.ntt.com/release_e/news07/0010/1017.html

8. NTT Com to Pilot Test Mobile Fragrance Communication Service, http: / /www.ntt.com/release_e/news08/0004/0407.html

9. Kaye, J.: Making Scents: aromatic output for HCI. Interactions 11(1), 48-61 (2004)

10. Edge Review: DigiScent Ismell, http: / / www . edgereview. com/ataglance. $\mathrm{cfm}$ ? category=Edge\&ID=136

11. http://www.osmooze.com/

12. Yamada, T., Tanikawa, T., Hirota, K., Hirose, M.: Wearable olfactory display: Using odor in outdoor environment. In: Proc. VR 2006, p. 199 (2006)

13. Kawasaki, T., Nakajima, M., Tonoike, M.: Aroma Science Series 21 Characteristics andAnalytical Estimation of Smell Material. Fragrance Journal Ltd (2003) (in Japanese)

14. Term and Commentary of Odor, Odor Control Association of Japan (2003) (in Japanese)

15. Sato, J., Ohtsu, K., Kadowaki, A., Bannai, Y., Okada, K.: Scent Presentation Technique by Pulse Ejection to avoid Olfactory Adaptation. Information Processing Society of Japan 49(8), 2922-2929 (2008)

16. Kadowaki, A., Sato, J., Ohtsu, K., Bannai, Y., Okada, K.: Measurement of Olfactory Responses to Odor Stimulation Switched in Single Breath. Journal of Japan Association on Odor Environment 40(1), 1-10 (2009) (in Japanese)

17. Ohtsu, K., Sato, J., Bannai, Y., Okada, K.: Scent Presentation Technique to Enable a Creating of Dynamic Perspective. Information Processing Society of Japan 50(4), 14351443 (2009) 